\title{
Front Matter: Volume 8352
}

, "Front Matter: Volume 8352," Proc. SPIE 8352, 28th European Mask and Lithography Conference, 835201 (10 May 2012); doi: 10.1117/12.945763

SPIE Event: 28th European Mask and Lithography Conference (EMLC 2012), 2012, Dresden, Germany 


\title{
PROCEEDINGS OF SPIE
}

\section{8th European Mask and Lithography Conference}

\author{
Uwe F.W. Behringer \\ Wilhelm Maurer \\ Editors
}

17-18 January 2012

Dresden, Germany

Organized by

VDE/VDI GMM-The Society for Microelectronics,

Micro- and Precision Engineering (Germany)

Published by

SPIE 
The papers included in this volume were part of the technical conference cited on the cover and title page. Papers were selected and subject to review by the editors and conference program committee. Some conference presentations may not be available for publication. The papers published in these proceedings reflect the work and thoughts of the authors and are published herein as submitted. The publisher is not responsible for the validity of the information or for any outcomes resulting from reliance thereon.

Please use the following format to cite material from this book:

Author(s), "Title of Paper," in 28th European Mask and Lithography Conference, edited by Uwe F.W. Behringer, Wilhelm Maurer, Proceedings of SPIE Vol. 8352 (SPIE, Bellingham, WA, 2012) Article CID Number.

ISSN 0277-786X

ISBN 9780819490308

Published by

SPIE

P.O. Box 10, Bellingham, Washington 98227-0010 USA

Telephone +1 3606763290 (Pacific Time) · Fax +1 3606471445

SPIE.org

Copyright (C) 2012, Society of Photo-Optical Instrumentation Engineers

Copying of material in this book for internal or personal use, or for the internal or personal use of specific clients, beyond the fair use provisions granted by the U.S. Copyright Law is authorized by SPIE subject to payment of copying fees. The Transactional Reporting Service base fee for this volume is $\$ 18.00$ per article (or portion thereof), which should be paid directly to the Copyright Clearance Center (CCC), 222 Rosewood Drive, Danvers, MA 01923. Payment may also be made electronically through CCC Online at copyright.com. Other copying for republication, resale, advertising or promotion, or any form of systematic or multiple reproduction of any material in this book is prohibited except with permission in writing from the publisher. The CCC fee code is 0277-786X/12/\$18.00.

Printed in the United States of America.

Publication of record for individual papers is online in the SPIE Digital Library.

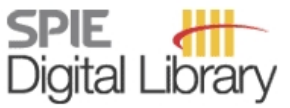

SPIEDigitalLibrary.org

Paper Numbering: Proceedings of SPIE follow an e-First publication model, with papers published first online and then in print and on CD-ROM. Papers are published as they are submitted and meet publication criteria. A unique, consistent, permanent citation identifier (CID) number is assigned to each article at the time of the first publication. Utilization of CIDs allows articles to be fully citable as soon as they are published online, and connects the same identifier to all online, print, and electronic versions of the publication. SPIE uses a six-digit CID article numbering system in which:

- The first four digits correspond to the SPIE volume number.

- The last two digits indicate publication order within the volume using a Base 36 numbering system employing both numerals and letters. These two-number sets start with 00, 01, 02, 03, 04, $05,06,07,08,09,0 A, 0 B \ldots 0 Z$, followed by 10-1Z, 20-2Z, etc.

The CID number appears on each page of the manuscript. The complete citation is used on the first page, and an abbreviated version on subsequent pages. Numbers in the index correspond to the last two digits of the six-digit CID number. 


\title{
Contents
}

\author{
ix Conference Committees \\ xiii Foreword \\ Xv Cooperating Partners and Sponsors \\ xvii Best Poster from BACUS 2011 \\ Optimization of mask shot count using MB-MDP and lithography simulation [8166-110] \\ G. S. Chua, W. L. Wang, B. I. Choi, GLOBALFOUNDRIES Singapore (Singapore); Y. Zou, \\ C. Tabery, GLOBALFOUNDRIES Inc. (United States); I. Bork, T. Nguyen, A. Fujimura, D2S Inc. \\ (United States)
}

\section{PLENARY SESSION}

835202 Nanometer-level semiconductor imaging for micrometer-level MEMS (Keynote Paper) [8352-100]

B. J. Lin, Taiwan Semiconductor Manufacturing Co. Ltd. (Taiwan)

835203 Mask industry assessment trend analysis: 2012 (Invited Paper) [8352-36]

Y. D. Chan, SEMATECH North (United States)

\section{EUV LITHOGRAPHY AND MASK APPLICATION}

835204 Mask readiness for EUVL pilot line (Invited Paper) [8352-28]

N. Hayashi, T. Abe, T. Takikawa, Dai Nippon Printing Co., Ltd. (Japan)

835205 NXE:3100 full wafer imaging performance and budget verification [8352-09]

E. van Setten, K. van Ingen Schenau, M. O'Mahony, T. Hollink, F. Wittebrood, N. Davydova, M. Eurlings, K. Feenstra, J. Finders, ASML Netherlands B.V. (Netherlands); M. Dusa, ASML

(Belgium); S. Young, ASML Netherlands B.V. (Netherlands)

835207 A fast approach to model EUV mask 3D and shadowing effects [8352-23]

Y. Li, D. Peng, M. Satake, P. Hu, Luminescent Technologies, Inc. (United States)

835208 EUVL mask performance and optimization [8352-19]

N. Davydova, R. de Kruif, E. van Setten, ASML Netherlands B.V. (Netherlands); B. Connolly, Toppan Photomasks, Inc. (Germany); K. Mehagnoul, ASML Netherlands B.V. (Netherlands); J. Zimmerman, N. Harned, ASML (United States); F. Kalk, Toppan Photomasks, Inc. (United States) 
835209 AIMS D2DB simulation for DUV and EUV mask inspection [8352-18]

D. Peng, Y. Li, M. Satake, P. Hu, Luminescent Technologies, Inc. (United States); J. Chen, S. C. Hsu, R. Lai, C. S. Lin, L. C. C. Tuo, Taiwan Semiconductor Manufacturing Co. Ltd. (Taiwan)

8352 OA Correcting image placement errors using registration control (RegC) technology in the photomask periphery (Best Paper of EMLC 2012) [8352-11]

A. Cohen, Carl Zeiss SMS Ltd. (Israel); F. Lange, Advanced Mask Technology Ctr. GmbH Co. KG (Germany); G. Ben-Zvi, E. Graitzer, V. Dmitriev, Carl Zeiss SMS Ltd. (Israel)

\section{LITHOGRAPHY OPTIMIZATION}

8352 OB Optimization method of photolithography process by means of atomic force microscopy [8352-05]

A. Sierakowski, P. Janus, Institute of Electron Technology (Poland); D. Kopiec, K. Nieradka, Wrocław Univ. of Technology (Poland); K. Domanski, P. Grabiec, Institute of Electron Technology (Poland); T. Gotszalk, Wrocław Univ. of Technology (Poland)

8352 OC Investigation and mitigation of field-edge CDU fingerprint for Arfi lithography for 45-nm to sub-28-nm logic nodes [8352-10]

B. Le-Gratiet, STMicroelectronics (France); J. Finders, O. Mouraille, R. Queens, M. Escalante, B. Smeets, J. Beltman, ASML Netherlands B.V. (Netherlands); K. Jullian, STMicroelectronics (France)

8352 OD Double exposure as a method to correct on-wafer CD variations: a proposal [8352-32] A. Hotzel, H. Bald, GLOBALFOUNDRIES Dresden Module One LLC \& Co. KG (Germany)

\section{LITHOGRAPHY FOR MEMS}

8352 OE Lithographic aspects for the fabrication of BiCMOS embedded bio-MEMS and RF-MEMS [8352-04]

P. Kulse, M. Birkholz, K.-E. Ehwald, M. Kaynak, M. Wietstruck, J. Bauer, J. Drews, K. Schulz, IHP $\mathrm{GmbH}$ (Germany)

8352 OF MEMS: fabrication of cryogenic bolometers [8352-34]

J. Kunert, S. Anders, T. May, V. Zakosarenko, G. Zieger, Institute of Photonic Technology (Germany); E. Kreysa, Max Planck Institute for Radioastronomy (Germany); H.-G. Meyer, Institute of Photonic Technology (Germany) 
8352 OG Impact of reticle absorber on the imaging properties in Arfi lithography (Invited Paper) [8352-03]

J. Finders, O. Mouraille, A. Bouma, A. Ngai, K. Grim, J. van Praagh, C. Toma, ASML Netherlands B.V. (Netherlands); J. Miyazaki, ASML Japan Co., Ltd. (Japan); M. Higuchi, Y. Kojima, Toppan Printing Co., Ltd. (Japan); B. Connolly, Toppan Photomasks, Inc. (Germany); I. Englard, Y. Cohen, S. Mangan, M. Ben Yishai, Applied Materials, Inc. (Israel); K. Jullian, STMicroelectronics (France)

$8352 \mathrm{OH} \quad$ PSM and thin OMOG reticles aerial imaging metrology comparison study [8352-12] Y. Cohen, Applied Materials, Inc. (Israel); J. Finders, ASML Netherlands B.V. (Netherlands);

S. Mangan, Applied Materials, Inc. (Israel); I. Englard, Applied Materials B.V. (Netherlands); O. Mouraille, M. Janssen, ASML Netherlands B.V. (Netherlands); J. Miyazaki, ASML Japan Co., Ltd. (Japan); B. Connolly, Toppan Photomasks, Inc. (Germany); Y. Kojima, M. Higuchi, Toppan Printing Co., Ltd. (Japan)

\section{MASK DATA PREPARATION}

835201 Pointwise process proximity function calibration: PPFexplorer application results [8352-17] M. Krueger, EQUIcon Software GmbH Jena (Germany); M. Banasch, Vistec Electron Beam GmbH (Germany); R. Galler, D. Melzer, EQUIcon Software GmbH Jena (Germany); L. E. Ramos, Vistec Electron Beam GmbH (Germany); M. Suelzle, EQUlcon Software GmbH Jena (Germany); U. Weidenmueller, Vistec Electron Beam GmbH (Germany); U. Zeitner, Fraunhofer Institute for Applied Optics and Precision Engineering (Germany)

8352 0J Mask write time reduction: deployment of advanced approaches and their impact on established work models [8352-33]

S. F. Schulze, A. Elayat, T. Lin, E. Sahouria, Mentor Graphics Corp. (United States)

8352 OK Improvements on Corner2, a lossless layout image compression algorithm for maskless lithography systems [8352-30]

J. Yang, Univ. of Michigan (United States); S. A. Savari, Texas A\&M Univ. (United States)

\section{EMERGING LITHOGRAPHY}

8352 OM Enhanced e-beam pattern writing for nano-optics based on character projection [8352-15] E.-B. Kley, H. Schmidt, Friedrich-Schiller-Univ. Jena (Germany); U. Zeitner, Fraunhofer Institute for Applied Optics and Precision Engineering (Germany); M. Banasch, B. Schnabel, Vistec Electron Beam GmbH (Germany)

8352 ON A novel tool for frequency assisted thermal nanoimprint (T-NIL) [8352-06] A. Mayer, K. Dhima, S. Möllenbeck, S. Wang, H.-C. Scheer, Bergische Univ. Wuppertal (Germany) 
835200 Nanoimprint activities in Austria in the research project cluster NILaustria [8352-21] M. Mühlberger, H. Fachberger, I. Bergmair, M. Rohn, B. Dittert, R. Schöftner, PROFACTOR GmbH (Austria); T. Rothländer, D. Nees, U. Palfinger, A. Haase, A. Fian, JOANNEUM RESEARCH Forschungsgesellschaft mbH (Austria); M. Knapp, OnkoTec Gmbh (Austria); C. Preininger, AIT Austrian Institute of Technology GmbH (Austria); G. Kreindl, M. Kast, EV Group (Austria); T. Fromherz, Johannes Kepler Univ. Linz (Austria)

8352 OP Phase-shift at subwavelength holographic lithography (SWHL) [8352-02]

M. V. Borisov, D. A. Chelyubeev, V. V. Chernik, A. A. Gavrikov, D. Yu. Knyazkov, P. A. Mikheev, V. I. Rakhovsky, A. A. Shamaev, Nanotech SWHL (Russian Federation)

MASK HANDLING, CLEANING, AND HAZE

$83520 Q \quad$ High quality mask storage in an advanced Logic-Fab [8352-27]

C. Jähnert, S. Fritsche, Infineon Technologies Dresden GmbH (Germany)

8352 OR Through pellicle management of haze formation in a wafer fabrication environment [8352-07]

A. Figliolini, M. Archuletta, J. LeClaire, D. Brinkley, D. Doerr, R. White, R. Bozak, D. A. Lee, RAVE LLC (United States)

8352 OS Cleaning aspects of material choice for high end mask manufacturing [8352-16] P. Nesladek, Advanced Mask Technology Ctr. GmbH Co. KG (Germany); S. Osborne, Osborne Enterprise (Germany); S. Rümmelin, Advanced Mask Technology Ctr. GmbH Co. KG (Germany)

8352 OT The effect of puddle megasonic cleaning for advanced photomask with subresolution assist features (SRAFs) [8352-20]

M.-C. Chen, H.-J. Yang, C.-R. Tseng, Taiwan Mask Corp. (Taiwan)

\section{EUV MASK DEFECT MANAGEMENT}

8352 OU Integrated cleaning and handling automation of NXE3100 reticles (Invited Paper) [8352-31] R. Jonckheere, IMEC (Belgium); T. Waehler, HamaTech APE GmbH \& Co. KG (Germany); B. Baudemprez, IMEC (Belgium); U. Dietze, SUSS MicroTec Inc. (United States); P. Dress, O. Brux, HamaTech APE GmbH \& Co. KG (Germany); K. Ronse, IMEC (Belgium)

$8352 \mathrm{OV}$ Towards the optical inspection sensitivity optimization of EUV masks and EUVL-exposed wafers [8352-29]

U. Okoroanyanwu, GLOBALFOUNDRIES Inc. (Germany); J. Heumann, Advanced Mask Technology Ctr. GmbH Co. KG (Germany); X. Zhu, C. H. Clifford, F. Jiang, P. Mangat, GLOBALFOUNDRIES Inc. (United States); R. Ghaskadvi, KLA-Tencor Corp. (United States); E. Mohn, R. Moses, Advanced Mask Technology Ctr. GmbH Co. KG (Germany); O. Wood, GLOBALFOUNDRIES Inc. (United States); H. Rolff, T. Schedel, R. Cantrell, P. Nesladek, Advanced Mask Technology Ctr. GmbH Co. KG (Germany); N. LiCausi, X. Cai, W. Taylor, J. Schefske, GLOBALFOUNDRIES Inc. (United States); M. Bender, Advanced Mask Technology Ctr. GmbH Co. KG (Germany); N. Schmidt, KLA-Tencor Germany (Germany)

8352 OW EUV mask defects and their removal [8352-37]

A. Rastegar, V. Jindal, SEMATECH North (United States) 
8352 OX EUVL defect printability: an industry challenge [8352-26]

H. J. Kwon, R. Teki, J. Harris-Jones, A. Cordes, SEMATECH North (United States)

8352 OY Advanced metrology techniques for the characterization of EUV mask blank defects [8352-24]

J. Harris-Jones, V. Jindal, C. C. Lin, T. Chakraborty, E. Stinzianni, R. Teki, H. J. Kwon, SEMATECH North (United States)

Author Index 
Downloaded From: https://www.spiedigitallibrary.org/conference-proceedings-of-spie on 26 Apr 2023

Terms of Use: https://www.spiedigitallibrary.org/terms-of-use 


\title{
Conference Committee
}

\author{
Members of the International Steering Committee and the International \\ Program Committee* of the EMLC 2012
}

Conference Chair

U. F.W. Behringer*, UBC Microelectronics (Germany)

Conference Cochairs

C. Gale*, Applied Materials (Germany)

N. Hayashi*, Dai Nippon Printing Company Ltd. (Japan)

Program Chairs

W. Maurer*, Infineon Technologies AG (Germany)

J. Finders*, ASML Netherlands B.V. (Netherlands)

R. Seltmann*, GLOBALFOUNDRIES (Germany)

Members
M. Arnz*, Carl Zeiss SMT AG (Germany)
E. Baracchi, STMicroelectronics (Italy)
J. Bauer*, IHP GmbH (Germany)
C. Blaesing, Carl Zeiss SMS GmbH (Germany)
P. Chen*, Taiwan Mask Corporation (Taiwan)
B. Connolly*, Toppan Photomasks Inc. (Germany)
R. Engelstad*, University of Wisconsin, Madison (United States)
J. Finders*, ASML Netherlands B.V. (Netherlands)
C. Gale*, Applied Materials (Germany)
B. Grenon, Grenon Consulting, Inc. (United States)
N. Hayashi*, Dai Nippon Printing Company Ltd. (Japan)
R. Jonckheere*, IMEC (Belgium)
B. Lauche, Photronics MZD GmbH, Dresden (Germany)
H. Loeschner*, IMS Nanofabrication AG (Austria)
W. Maurer*, Infineon Technologies AG (Germany)
C. Progler, Photronics Inc. (United States)
E. Rausa*, Plasma-Therm LLC (United States)
D. J. Resnick*, Molecular Imprints (United States)
K.-D. Röth*, KLA-Tencor Germany (Germany)
C. Romeo*, Numonyx (Italy)
H.-C. Scheer*, Bergische Universität Wuppertal (Germany)
T. Scherübl*, Carl Zeiss SMS GmbH (Germany) 
R. Schnabel*, VDE/VDI-GMM (Germany)

S. Schulze*, Mentor Graphics Corporation (United States)

R. Seltmann*, GLOBALFOUNDRIES, Inc. (Germany)

M. Staples, GLOBALFOUNDRIES, Inc. (Germany)

I. Stolberg*, Vistec Electron Beam GmbH Jena (Germany)

S. Tedesco*, CEA-LETI (France)

M. Tissier*, Toppan Photomasks S.A. (France)

J. Waelpoel*, ASML Netherlands B.V. (Netherlands)

G. Wenz*, Wenz Consulting (Germany)

J. Whittey*, KLA-Tencor Corporation (United States)

H. Wolf*, Photronics MZD GmbH (Germany)

S. Wurm*, SEMATECH North (United States)

L. Zurbrick*, Agilent Technologies (United States)

\section{Session Chairs}

1 Plenary Session I

W. Maurer*, Infineon Technologies AG (Germany)

R. Seltmann*, GLOBALFOUNDRIES, Inc. (Germany)

2 Plenary Session II

C. Gale*, Applied Materials (Germany)

N. Hayashi*, Dai Nippon Printing Company Ltd. (Japan)

3 EUV Lithography and Mask Application

T. Scherübl*, Carl Zeiss SMS GmbH (Germany)

B. Connolly*, Toppan Photomasks Inc. (Germany)

4 Mask Optimization

D. Farrar, HOYA Corporation (United Kingdom)

F. Goodwin, SEMATECH North (United States)

5 Lithography Optimization

J. Finders*, ASML Netherlands B.V. (Netherlands)

J. Whittey*, KLA-Tencor Corporation (United States)

$6 \quad$ Lithography for MEMS

J. Baver*, IHP GmbH (Germany)

U. F.W. Behringer*, UBC Microelectronics (Germany)

$7 \quad$ Mask Materials

H. Wolf*, Photronics MZD GmbH (Germany)

R. Seltmann*, GLOBALFOUNDRIES, Inc. (Germany)

8 Mask Data Preparation

I. Stolberg*, Vistec Electron Beam GmbH Jena (Germany)

W. Maurer*, Infineon Technologies AG (Germany) 
$9 \quad$ Emerging Lithography

H.-C. Scheer*, Bergische Universität Wuppertal (Germany)

S. Tedesco*, CEA-LETI (France)

10 Mask Handling, Cleaning, and Haze

N. Hayashi*, Dai Nippon Printing Company Ltd. (Japan)

R. Jonckheere*, IMEC (Belgium)

11 EUV Mask Defect Management

F. Goodwin, SEMATECH North (United States)

C. Gale*, Applied Materials (Germany)

12 Last Minute Submisssions

U. F.W. Behringer*, UBC Microelectronics (Germany) 
Downloaded From: https://www.spiedigitallibrary.org/conference-proceedings-of-spie on 26 Apr 2023

Terms of Use: https://www.spiedigitallibrary.org/terms-of-use 


\section{Foreword}

Welcome to the proceedings volume from the 28th European Mask and Lithography conference, EMLC2012, which was held 17-18 January 2012 at the Hilton Hotel in Dresden, Germany. On behalf of VDE/VDI-GMM, the sponsors, and the organizing committee, we are pleased to present to you these papers presented at EMLC2012.

Every year this conference brings together scientists, researchers, engineers, and technologists from research institutes and companies from around the world to present innovations at the forefront of mask lithography and mask technology. The two-day conference is dedicated to the science, technology, engineering, and application of mask and lithography technologies and associated processes, giving an overview of the present status in mask and lithography technologies and the future strategy where mask producers and users have the opportunity of becoming acquainted with new developments and results. This year's sessions included: EUV Lithography and Masks Application, Mask Optimization, Lithography Optimization, Lithography for MEMS, Mask Materials, Mask Data Preparation, Emerging Lithography, Mask Handling, Cleaning, and Haze, and EUV Mask Defect Management. For post-deadline submissions there was the "Last Minute Session" at the end of the conference.

We are pleased to report that Martin Esser, President Silicon Saxony, Dresden, provided the opening comments. Our first keynote speaker was Dr. Burn Lin from TSMC, Taiwan. His presentation was entitled "Nanometer-level semiconductor for micrometer-level MEMS." Our second keynote speaker was Dr. Paul Chipman, Vice President, Global Strategy, Toppan Photomasks Inc., of Austin, TX, USA.

An annual fixture has been SEMATECH's Trend Analysis of their Mask industry Assessment, presented this year by David Chan from SEMATECH, Albany, New York, USA.

The Best Paper of EMLC2012 was "Correcting image placement errors by using registration control (RegC) technology in the photomask periphery," by Avi Cohen, et al, from Carl Zeiss SMS, Israel.

Parallel to the Conference Presentations, a Technical Exhibition took place on Tuesday and Wednesday where companies (mask suppliers, material suppliers, and equipment suppliers) showed off their companies and products. In order to foster the exchange between the conference attendees and the exhibitors, the exhibition area also served as the place for all coffee and lunch breaks. 
We hope that you enjoyed the Technical Sessions of the EMLC2012 as well as the Technical Exhibition, but also that you allowed yourself to visit Dresden, one of the most beautiful cities in Europe!

Please mark your calendar: EMLC2013 will move from January to June. So the next conference, EMLC2013, will be 25-27 June 2013 at the same location, the Hilton Hotel in Dresden, Germany.

Uwe F.W. Behringer 


\section{Cooperating Partners and Sponsors of the EMLC 2012}

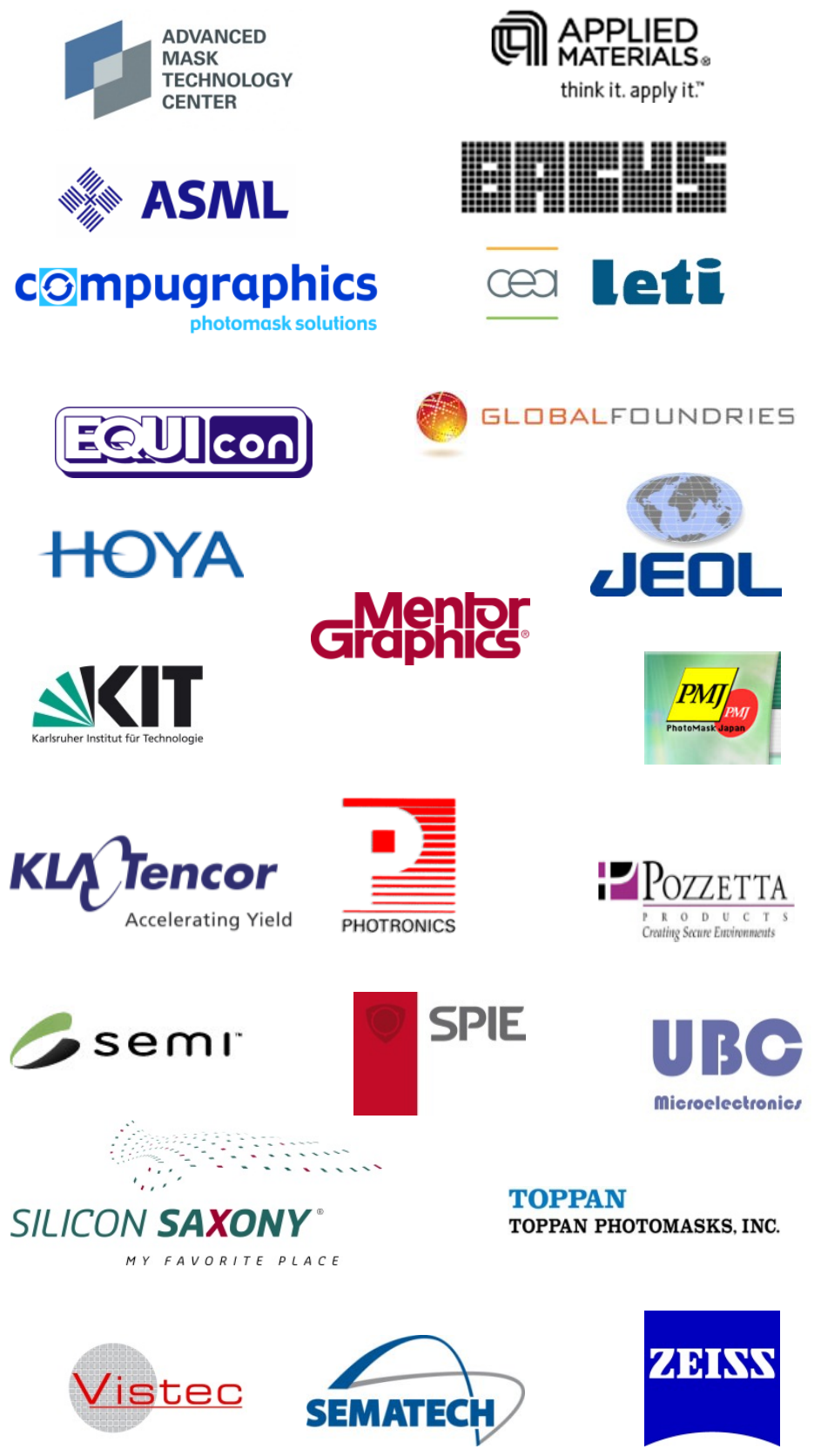


Downloaded From: https://www.spiedigitallibrary.org/conference-proceedings-of-spie on 26 Apr 2023

Terms of Use: https://www.spiedigitallibrary.org/terms-of-use 